\title{
Searching for BSM neutrino interactions in dark matter detectors
}

\author{
Jonathan M. Link ${ }^{a}$ and Xun-Jie $\mathbf{X} \mathbf{u}^{b}$ \\ ${ }^{a}$ Center for Neutrino Physics, Physics Department, Virginia Tech, \\ 850 West Campus Dr, Blacksburg, VA 24061, U.S.A. \\ ${ }^{b}$ Max-Planck-Institut für Kernphysik, \\ Postfach 103980, D-69029 Heidelberg, Germany \\ E-mail: jmlink@vt.edu, xunjie.xu@gmail.com
}

ABSTRACT: Neutrino interactions beyond the Standard Model (BSM) are theoretically well motivated and have an important impact on the future precision measurement of neutrino oscillation. In this work, we study the sensitivity of a multi-ton-scale liquid Xenon dark matter detector equipped with an intense radioactive neutrino source to various BSM neutrino-electron interactions. We consider the conventional Non-Standard Interactions (NSIs), other more generalized four-fermion interactions including scalar and tensor forms, and light-boson mediated interactions. The work shows that with realistic experimental setups, one can achieve unprecedented sensitivity to these BSM neutrino-electron interactions.

KEywords: Beyond Standard Model, Neutrino Physics

ARXiv EPrint: 1903.09891 


\section{Contents}

1 Introduction 1

2 Event rates 2

3 Measurement of the SM parameters $\quad 4$

4 BSM neutrino interactions $\quad 7$

4.1 Non-standard interactions 8

$\begin{array}{llr}4.2 & \text { SPVAT interactions } & 9\end{array}$

4.3 Light $Z^{\prime} \quad 12$

$\begin{array}{llr}5 & \text { Conclusion } & 14\end{array}$

$\begin{array}{ll}\text { A Geometrical calculation of } \boldsymbol{r}_{\text {avg }} & 15\end{array}$

\section{Introduction}

The existence of small neutrino masses is calling for new physics. It is fairly reasonable to speculate that the interactions of neutrinos may also go beyond the Standard Model (BSM). Searching for BSM neutrino interactions is of increasing importance since they could make a considerable impact on future precision oscillation measurements. For example, it is known that the Non-Standard Interactions [1-4] of neutrinos may affect the measurement of $\delta_{C P}$ in long-baseline oscillation experiments [5-12]. Moreover, due to various well-known problems of the SM, theorists have for decades been looking for more satisfactory theories. As neutrino masses and mixing have become established, precision measurement of neutrino interactions are increasingly important in directing theoretical exploration.

Actually, if BSM neutrino interactions exist, charged leptons such as the electron are very likely to participate in these interactions as well, since neutrinos and charged leptons are embedded in $\mathrm{SU}(2)_{L}$ doublets in the $\mathrm{SM}$, and new interactions are generally expected to couple with the whole doublet. Many BSM theories such as Type II seesaw models [1318], left-right symmetric models [19-21], and the various U(1) extensions [22-28], are some typical examples which introduce new interactions between neutrinos and charged leptons.

Therefore, searching for neutrino-electron BSM interactions is theoretically highly motivated and has been extensively studied in the literature [29-44]. So far the most precise measurement for $\nu_{\mu}\left(\bar{\nu}_{\mu}\right)$-e elastic scattering, ${ }^{1}$ comes from the CHARM II experiment $[45,46]$, which achieved a precision of $3 \%$. For $\nu_{e}-e$ and $\bar{\nu}_{e^{-e}}$ scattering, the best

\footnotetext{
${ }^{1}$ Quasi-elastic scattering processes such as $\nu_{\mu(\tau)}+e \rightarrow \mu(\tau)+\nu_{e}$ have little potential of finding new physics since the corresponding charged current interactions have been very precisely determined by $\mu$ and $\tau$ decays.
} 
measurements come from LSND [47] and TEXONO [30] respectively, with only $20 \%$ precision. Recently there have been proposals [48-52] to using dark matter detectors with intense radioactive neutrino sources to measure neutrino scattering, that could lead to significant improvements. In particular, ton-scale liquid xenon (LXe) detectors [53-55] equipped with state-of-the-art ${ }^{51} \mathrm{Cr}$ sources [56] (referred to as ${ }^{51} \mathrm{Cr}$-LXe throughout this paper), with their combination of a large mono-energetic $\nu_{e}$ flux, high electron density, and low backgrounds, offer a path to reaching unprecedented precision.

In this paper, we study the potential of ${ }^{51} \mathrm{Cr}$-LXe experiments for probing BSM neutrino interactions. Our previous study [52] showed that the combination of a $5 \mathrm{MCi}{ }^{51} \mathrm{Cr}$ source and a 6 ton LXe detector could generate the tightest terrestrial constraint on neutrino magnetic moments, and competitive sensitivity to sterile neutrino oscillation. Therefore, we expect similar setups would have excellent sensitivity to a wide range of BSM neutrino interactions, including Non-Standard Interactions (NSIs), general four-fermion effective interactions (e.g., including tensor and scalar forms), and light and weakly coupled mediators (e.g. dark photons). The new physics scenarios considered in this work can also be tested in some other low-energy processes such as coherent elastic neutrinonucleus scattering $(\mathrm{CE} \nu \mathrm{NS})$ and atomic parity violation $(\mathrm{APV})$ - for a recent study, see ref. [57]. The main difference is that $\mathrm{CE} \nu \mathrm{NS}$ and APV test interactions between leptons (neutrinos or electrons) and quarks, while $\nu$-e scattering experiments test purely leptonic interactions between neutrinos and electrons. Although phenomenologically they are different, theoretically these interactions would be very closely correlated in some models. So combining both types of low-energy measurements could provide a complementary test of BSM theories.

The paper is organized as follows. In section 2, we introduce the basic experimental setup and evaluate the event rates of signals and backgrounds for several configurations. In section 3, we study the precision measurement of the SM parameters relevant to neutrino interactions. In section 4, we include sensitivity studies for several types of BSM neutrino interactions which are widely discussed in the literature. Finally, we conclude and summarize the results in section 5 .

\section{Event rates}

Let us consider a general ${ }^{51} \mathrm{Cr}$-LXe experiment and evaluate the event numbers of $\nu$-e scattering. In the ${ }^{51} \mathrm{Cr}$-LXe experiment, the number of $\nu$-e scattering events appearing in the infinitesimal volume $d V$ around position $\vec{r} \equiv(x, y, z)$ in the detector, during the time interval $t$ to $t+d t$, with the recoil energy from $T$ to $T+d T$ can be evaluated as

$$
d N=d V n_{e} d t d T\left[\int \phi\left(\vec{r}, t, E_{\nu}\right) \frac{d \sigma}{d T}\left(T, E_{\nu}\right) d E_{\nu}\right],
$$

where $n_{e}, \phi, \frac{d \sigma}{d T}$, and $E_{\nu}$ denote the electron number density, the neutrino flux, the differential cross section, and the neutrino energy respectively.

For a point-like radioactive source, $\phi$ has the following spatial and temporal dependence:

$$
\phi\left(\vec{r}, t, E_{\nu}\right)=\phi_{0} R_{\mathrm{Cr} 51}^{0} \frac{1 \mathrm{~m}^{2}}{r^{2}} e^{-t / \tau} f\left(E_{\nu}\right)
$$




\begin{tabular}{|lcccccc|}
\hline & $R_{\mathrm{Cr} 51}^{0}$ & $\Delta t$ & $\left\langle R_{\mathrm{Cr} 51}\right\rangle$ & background & $r_{\text {avg }}$ & $V n_{e}$ \\
\hline Configuration A & $5 \mathrm{MCi}{ }^{51} \mathrm{Cr}$ & 100 days & $1.83 \mathrm{MCi}$ & normal & $1.63 \mathrm{~m}$ & $1.5 \times 10^{30}$ \\
Configuration B & $5 \mathrm{MCi}{ }^{51} \mathrm{Cr}$ & 50 days & $2.85 \mathrm{MCi}$ & ${ }^{136}$ Xe depleted & $1.63 \mathrm{~m}$ & $1.5 \times 10^{30}$ \\
Configuration C & $10 \mathrm{MCi}{ }^{51} \mathrm{Cr}$ & 50 days & $5.71 \mathrm{MCi}$ & ${ }^{136}$ Xe depleted & $1.63 \mathrm{~m}$ & $1.5 \times 10^{30}$ \\
\hline
\end{tabular}

Table 1. Configurations of the ${ }^{51} \mathrm{Cr}-\mathrm{LX}$ experiment considered in this work.

where

$$
\phi_{0}=2.94 \times 10^{15} \text { neutrinos } /\left(\mathrm{MCi} \mathrm{m}^{2} \mathrm{~s}\right),
$$

is the neutrino flux at 1 meter from a $1 \mathrm{MCi}$ radioactive source; $\tau=39.96$ days is the mean lifetime of ${ }^{51} \mathrm{Cr} ; R_{\mathrm{Cr} 51}^{0}$ is the initial $(t=0)$ radioactivity; and $f\left(E_{\nu}\right)$ describes the spectral shape, normalized by $\int f\left(E_{\nu}\right) d E_{\nu}=1$. The neutrino spectrum of ${ }^{51} \mathrm{Cr}$ simply consists of mono-energetic neutrino emissions at $750 \mathrm{keV}(90 \%)$ and $430 \mathrm{keV}(10 \%)$ :

$$
f\left(E_{\nu}\right)=0.9 \delta\left(E_{\nu}-750 \mathrm{keV}\right)+0.1 \delta\left(E_{\nu}-430 \mathrm{keV}\right) .
$$

Since the source decays exponentially, it is useful to define a time-averaged activity,

$$
\left\langle R_{\mathrm{Cr} 51}\right\rangle \equiv \frac{R_{\mathrm{Cr} 51}^{0}}{\Delta t} \int_{0}^{\Delta t} d t e^{-t / \tau}=\frac{\tau R_{\mathrm{Cr} 51}^{0}}{\Delta t}\left[1-e^{-\Delta t / \tau}\right]
$$

relative to the initial activity, $R_{\mathrm{Cr} 51}^{0}$, and the exposure time, $\Delta t$. Likewise, an average distance between the detector and the source, $r_{\mathrm{avg}}$, can be defined as:

$$
\frac{1}{r_{\mathrm{avg}}^{2}} \equiv\left\langle\frac{1}{r^{2}}\right\rangle=\frac{1}{V} \int \frac{d V}{r^{2}}
$$

With these averaged values, it is equivalent use the following replacement in our analyses:

$$
\phi\left(\vec{r}, t, E_{\nu}\right) \rightarrow \phi_{0} \frac{1 \mathrm{~m}^{2}}{r_{\text {avg }}^{2}}\left\langle R_{\mathrm{Cr} 51}\right\rangle f\left(E_{\nu}\right) \equiv \phi_{\mathrm{avg}}\left(E_{\nu}\right),
$$

while collapsing the time and space integrals to the $\Delta t$ and the detector fiducial volume, $V$. Hence the number of events in the recoil energy bin $\left[T_{i}, T_{i}+\Delta T\right]$ can be written as

$$
N_{i}=V n_{e} \Delta t \int_{T_{i}}^{T_{i}+\Delta T} \Phi(T) d T,
$$

with

$$
\Phi(T) \equiv \int \phi_{\mathrm{avg}}\left(E_{\nu}\right) \frac{d \sigma}{d T}\left(T, E_{\nu}\right) d E_{\nu}
$$

where $\phi_{\text {avg }}\left(E_{\nu}\right)$ has been defined in eq. (2.7).

For a detector with a cylindrical fiducial region, $r_{\text {avg }}$ can be computed analytically (see appendix A). We take the same geometrical profile as ref. [52], i.e., the height $(h)$ and diameter $(d)$ of the cylinder are $h=d=1.38 \mathrm{~m}$, and the source is $L=1 \mathrm{~m}$ below the bottom. Using eq. (A.5), this profile has $r_{\text {avg }}=1.63 \mathrm{~m}$ and contains 6 tons of LXe, which corresponds to $V n_{e}=1.5 \times 10^{30}$ electrons. 


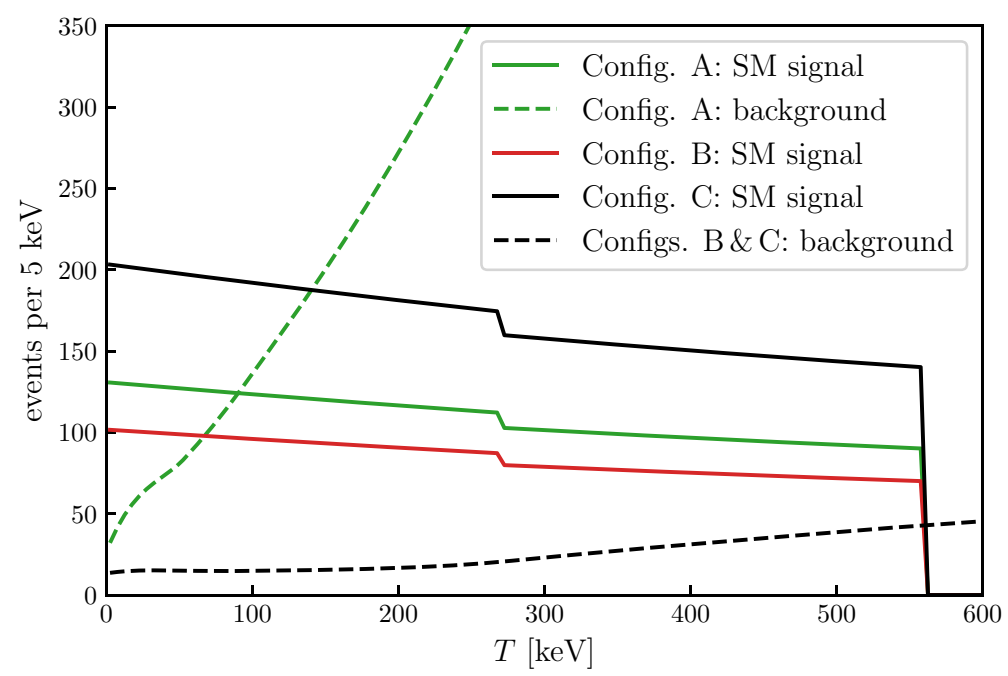

Figure 1. Signal compared to background in the ${ }^{51} \mathrm{Cr}-\mathrm{LX}$ experiment. Three configurations (A, $\mathrm{B}, \mathrm{C})$ are considered, with the details described in table 1. Configurations $\mathrm{B}$ and $\mathrm{C}$ have much larger signal/background ratios than A.

Plugging in the definition of $\phi_{\mathrm{avg}}$, the integral in eq. (2.9) works out as

$$
\Phi(T)=\frac{1 \mathrm{~m}^{2}}{r_{\mathrm{avg}}^{2}}\left\langle R_{\mathrm{Cr} 51}\right\rangle \phi_{0}\left[0.9 \frac{d \sigma}{d T}\left(T, E_{\nu}=750 \mathrm{keV}\right)+0.1 \frac{d \sigma}{d T}\left(T, E_{\nu}=430 \mathrm{keV}\right)\right] .
$$

In practical calculations, we also need the maximal recoil energy, $T_{\max }$, which is defined as the maximal recoil of electron that can be generated by a certain $E_{\nu}$ :

$$
T_{\max }=\frac{2 E_{\nu}^{2}}{2 E_{\nu}^{2}+m_{e}} \approx\left\{\begin{array}{ll}
559 \mathrm{keV} & \left(E_{\nu}=750 \mathrm{keV}\right) \\
270 \mathrm{keV} & \left(E_{\nu}=430 \mathrm{keV}\right)
\end{array} .\right.
$$

For $T>T_{\max }$ the corresponding $\frac{d \sigma}{d T}$ in eq. (2.10) is set to zero.

In table 1, we summarize useful quantities for the configurations considered in this work. The event numbers with the SM $\nu$-e elastic scatting cross section [58] are evaluated using eq. (2.8) and presented in figure 1. Regarding backgrounds, we assume a "normal" level [59], which includes the solar neutrino background and decays of embedded radioactive isotopes $\left({ }^{136} \mathrm{Xe},{ }^{85} \mathrm{Kr},{ }^{222} \mathrm{Rn}\right.$, etc.), for configuration A; and a " ${ }^{136} \mathrm{Xe}$ depleted" level, which consists of the normal solar background with only $10 \%$ of the normal level from radioactive decays, for configurations $\mathrm{B}$ and $\mathrm{C}$. In configurations $\mathrm{B}$ and $\mathrm{C}$, the background rate is further decreased by halving the exposure time, $\Delta t$. Finally, in configuration $\mathrm{C}$, the signalto-noise ratio is further increased by the use of a more intense source.

\section{Measurement of the SM parameters}

The study of neutrino-electron scattering has played an important role in the precision electroweak tests of the SM - see Chapter 10 of PDG [60]. For instance, the CHARM II 
experiment, which has performed the most precise measurement of $\nu_{\mu}-e$ and $\bar{\nu}_{\mu}-e$ scattering thus far, has determined the electroweak mixing angle, $\sin ^{2} \theta_{W}$, to be:

$$
\sin ^{2} \theta_{W}=0.2324 \pm 0.0083, \quad(\text { CHARM II, 1994), }
$$

with about $3 \%$ precision [46]. In comparison $\stackrel{(-)}{\nu_{e}}$ scattering experiments have not yet achieved such precision. The best measurements of $\nu_{e^{-}}$and $\bar{\nu}_{e^{-e}}$ scattering come from LSND [47] and TEXONO [30] respectively:

$$
\sin ^{2} \theta_{W}=\left\{\begin{array}{ll}
0.248 \pm 0.051 & \left(\nu_{e}-e \mathrm{LSND}\right) \\
0.251 \pm 0.031 \pm 0.024 & \left(\bar{\nu}_{e}-e \mathrm{TEXONO}\right)
\end{array} .\right.
$$

We will show that a ${ }^{51} \mathrm{Cr}$-LXe experiment could provide a superior precision measurement of $\sin ^{2} \theta_{W}$, which not only exceeds that of these aforementioned experiments, but also reverses the current situation in which $\stackrel{(-)}{\nu})$ scattering is less precise than $\stackrel{(-)}{\nu}$ scattering.

In the SM, the low energy interactions of neutrinos and electrons can be described by the effective Lagrangian

$$
\mathcal{L}_{\mathrm{NC}+\mathrm{CC}} \supset \frac{G_{F}}{\sqrt{2}} \bar{\nu}_{e} \gamma^{\mu}\left(1-\gamma^{5}\right) \nu_{e} \bar{\psi}_{e} \gamma^{\mu}\left[g_{V}-g_{A} \gamma^{5}\right] \psi_{e},
$$

where, for $\nu_{e^{-}}$scattering, $g_{V}^{e}$ and $g_{A}^{e}$ are given at the tree level by

$$
g_{V}^{e}=2 \sin ^{2} \theta_{W}+\frac{1}{2} \text { and } g_{A}^{e}=\frac{1}{2}
$$

which includes both the neutral current (NC) and the charged current (CC) contributions. At the tree level, the differential cross section for $\nu$-e scattering is given by [58]:

$$
\frac{d \sigma}{d T}=\frac{m_{e} G_{F}^{2}}{2 \pi}\left[g_{1}^{2}+g_{2}^{2}\left(1-\frac{T}{E_{\nu}}\right)^{2}-g_{1} g_{2} \frac{m_{e} T}{E_{\nu}^{2}}\right],
$$

where $T$ is the electron's recoil energy and

$$
g_{1} \equiv g_{V}+g_{A} \text { and } g_{2} \equiv g_{V}-g_{A} .
$$

The experimental value of $\sin ^{2} \theta_{W}$ is well determined at the $Z$-pole and found to be 0.23122 (defined in the Modified Minimal Subtraction ( $\overline{\mathrm{MS}}$ ) scheme [60]). At low energies $(\lesssim 0.1 \mathrm{GeV})$, its value can be extrapolated theoretically using renormalization group equation (RGE) running [61]:

$$
\sin ^{2} \theta_{W} \approx 0.23865 \quad \text { (low energy limit). }
$$

Including the radiative corrections, the expressions of $g_{V}$ and $g_{A}$ should also be modified [62]:

$$
g_{V}^{e} \approx 2.00128 \sin ^{2} \theta_{W}+0.4828 \text { and } g_{A}^{e} \approx 0.4936 .
$$

Taking $\sin ^{2} \theta_{W}$ as a varying parameter, one can measure it in the ${ }^{51} \mathrm{Cr}$-LXe experiment, as a low-energy precision test of the SM. The simulated data is generated assuming the 


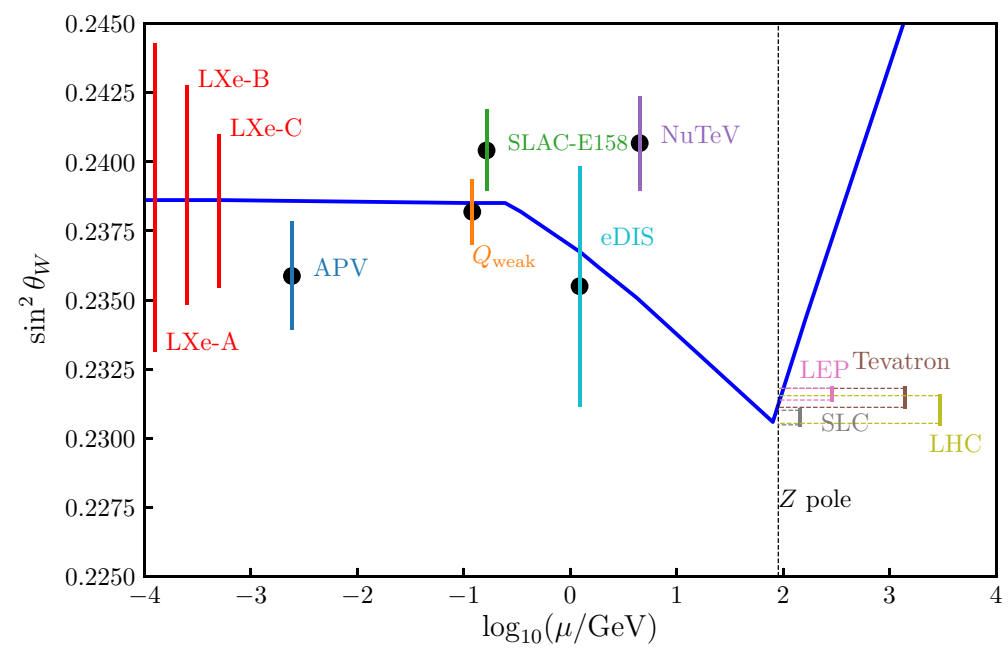

Figure 2. Measurement of $\sin ^{2} \theta_{W}$ at different energy scales $(\mu)$. The red error bars represent the precision of LXe-A, LXe-B, and LXe-C expected from this work. The other measurements are from figure 10.2 of the PDG [60]. Measurements from earlier neutrino elastic scattering experiments do not have sufficient precision to be included in this plot.

\begin{tabular}{|cccc|}
\hline & Configuration A & Configuration B & Configuration C \\
\hline precision of $\sin ^{2} \theta_{W}$ & $0.23865 \pm 0.0055$ & $0.23865_{-0.0037}^{+0.0041}$ & $0.23865_{-0.0031}^{+0.0023}$ \\
relative precision & $\pm 2.3 \%$ & ${ }_{-1.6 \%}^{+1.7 \%}$ & ${ }_{-1.3 \%}^{+1.0 \%}$ \\
\hline
\end{tabular}

Table 2. Precision of the $\sin ^{2} \theta_{W}$ measurement in the three ${ }^{51} \mathrm{Cr}$-LXe configurations considered.

numerical values in eqs. (3.7) and (3.8), and including the backgrounds shown in figure 1. Then we perform a $\chi^{2}$-fit on the data to obtain the $1 \sigma$ confidence level (CL) of $\sin ^{2} \theta_{W}$. The results are presented in figure 2 and table 2 . Comparing these results with the current stateof-the-art from CHARM II, LSND, and TEXONO, we see that the ${ }^{51} \mathrm{Cr}$-LXe experiment is able to reach unprecedented precision on $\sin ^{2} \theta_{W}$ as measured by $\nu$-e scattering, even in the least sensitive configuration that we have considered.

In figure 2, we show the role of ${ }^{51} \mathrm{Cr}$-LXe measurements in probing the RGE running of $\sin ^{2} \theta_{W}$. So far, the most precise measurements of $\sin ^{2} \theta_{W}$ are from colliders, including LEP, LHC, SLC, and Tevatron. All the colliders essentially measure the value of $\sin ^{2} \theta_{W}$ only at the $Z$-pole scale. Other measurements, including the weak charge of protons $\left(Q_{\text {weak }}\right)$, Atomic Parity Violation (APV), and electron deep-inelastic scattering (eDIS), are located at various lower energy scales. This plot shows that a precision probe of the RGE running of $\sin ^{2} \theta_{W}$, is still needed at the lowest energies, which a ${ }^{51} \mathrm{Cr}$-LXe measurement (marked in red as LXe-A, B and C) would provide.

The ${ }^{51} \mathrm{Cr}-\mathrm{LX}$ experiment would also provide a high precision measurement of the weak coupling parameters $g_{V}^{e}$ and $g_{A}^{e}$. We performed a simultaneous $\chi^{2}$-fit of $g_{V}^{e}$ and $g_{A}^{e}$ based on eqs. (3.5) and (3.6). Figure 3 shows the fit result in the $\left(g_{V}^{e}, g_{A}^{e}\right)$ plane, and compares it with the measurements from TEXONO, LSND, and CHARM II, where for $\stackrel{(-)}{\nu}-e$ scattering measurements of CHARM II, the weak coupling constants are converted 


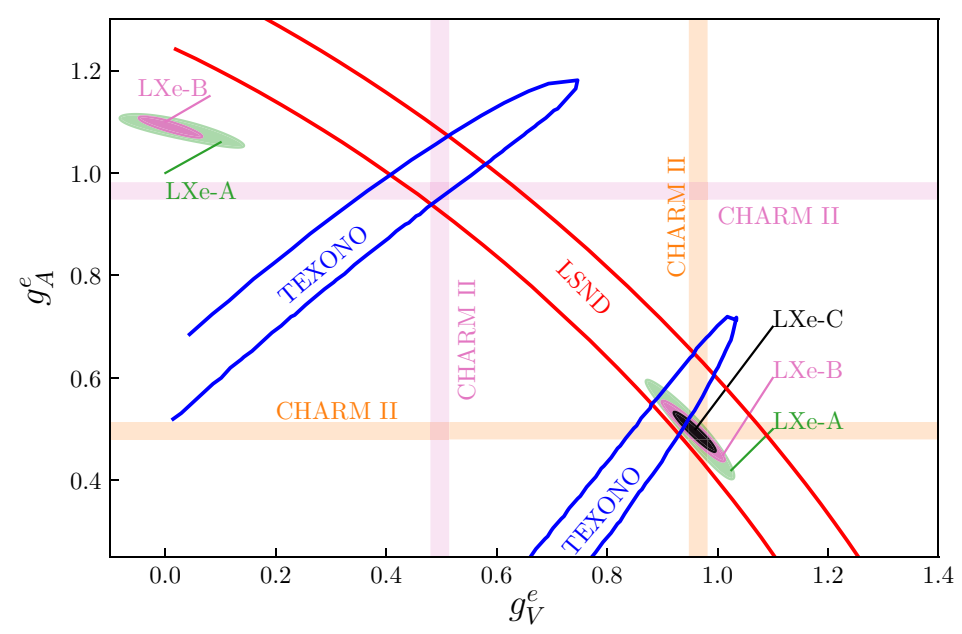

Figure 3. Sensitivity of ${ }^{51} \mathrm{Cr}$-LXe experiment to weak couplings parameters, $g_{V}^{e}$ and $g_{A}^{e}$, compared to existing constraints. The solid blobs represent the expected constraints, centered on the SM values, for the LXe-A, LXe-B, and LXe-B configurations in this work. Prior constraints from TEXONO (blue) and LSND (red) are taken from figure 10.1 of PDG [60], and the CHARM II constraint comes from ref. [46].

using $g_{V}^{e}=g_{V}^{\mu}+1$ and $g_{A}^{e}=g_{A}^{\mu}+1$. Due to low statistics, TEXONO and LSND appear as long bands, which are mainly determined by the total event numbers with little impact from spectral information. In other words, TEXONO and LSND effectively measured only the total cross section since their low-statistics measurements were not sensitive to spectral distortions from variations of $g_{V}$ and $g_{A}$. The CHARM II measurement, which has much higher statistics, gives $g_{V}^{\mu}=-0.035 \pm 0.017$ and $g_{A}^{\mu}=-0.503 \pm 0.017$ (see the overlap of two orange bands). However, since the neutrino energy is much higher than the electron mass in CHARM II, it also has some discrete parameter degeneracy - the cross section is approximately invariant when $\left(g_{V}^{\mu}, g_{A}^{\mu}\right) \rightarrow\left(g_{A}^{\mu}, g_{V}^{\mu}\right)$ and exactly invariant under $\left(g_{V}^{\mu}, g_{A}^{\mu}\right) \rightarrow\left(-g_{V}^{\mu},-g_{A}^{\mu}\right)$. For the ${ }^{51} \mathrm{Cr}$-LXe experiment, depending on the signal/background ratio, similar situation may appear as well. This can be understood by reformatting the cross section in powers of $T / E_{\nu}$ :

$$
\frac{d \sigma}{d T}=\frac{m_{e} G_{F}^{2}}{2 \pi}\left[\left(g_{1}^{2}+g_{2}^{2}\right)-\left(2 g_{2}^{2}+g_{1} g_{2} \frac{m_{e}}{E_{\nu}}\right) \frac{T}{E_{\nu}}+g_{2}^{2}\left(\frac{T}{E_{\nu}}\right)^{2}\right],
$$

which implies that for low recoil energies $\left(T / E_{\nu} \ll 1\right)$ we essentially measure only $g_{1}^{2}+g_{2}^{2}$, while higher energy recoils are also sensitive to $2 g_{2}^{2}+g_{1} g_{2} \frac{m_{e}}{E_{\nu}}$. With these two factors determined, $\left(g_{1}, g_{2}\right)$ would be approximately known up to some discrete ambiguities. This explains why configurations $\mathrm{A}$ and $\mathrm{B}$ have two separate low- $\Delta \chi^{2}$ regions, while for configuration $\mathrm{C}$, this degeneracy is broken by the higher signal/background measurement, which is also sensitive to the quadratic term in $T / E_{\nu}$.

\section{BSM neutrino interactions}

In many BSM theories, neutrinos have new interactions, which can be categorized into two types, (i) interactions mediated by heavy particles, and (ii) interactions mediated by light particles. 

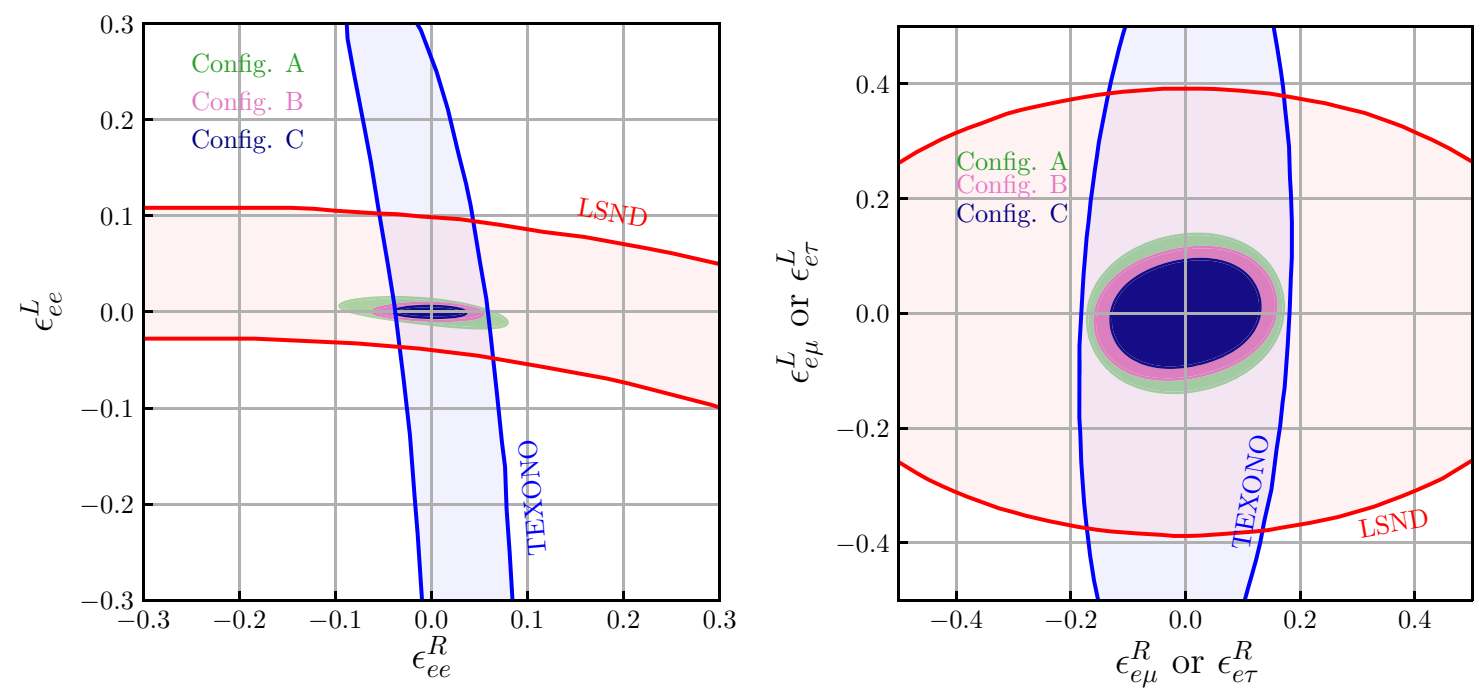

Figure 4. Sensitivity at $90 \% \mathrm{CL}$ of a ${ }^{51} \mathrm{Cr}$-LXe experiment on the NSI parameters, compared to the existing bounds from the LSND and TEXONO experiments [87].

For heavy mediators, one can integrate them out and study the effective four-fermion interactions. As a model-independent approach, one can write down all possible effective four-fermion interactions of neutrinos with electrons (as we are considering elastic neutrino-electron scattering). The most extensively studied effective interactions are the Non-Standard Interactions (NSIs, reviewed in [1-4]) which lead to interesting phenomenology in neutrino oscillations. In addition, one can consider the most general four-fermion operators with all possible Lorentz invariant forms considered, including Scalar, Pseudoscalar, Vector, Axialvector, and Tensor (SPVAT) interactions [29, 33, 35, 63-72]. We will study the sensitivity of a ${ }^{51} \mathrm{Cr}-\mathrm{LXe}$ experiment to both frameworks.

For light mediators, the interactions can be much more multifarious. As a demonstration of light mediator sensitivity, we will study a light $Z^{\prime}$ with a very weak gauge coupling. Such a scenario is particular important for dark matter studies [73-84] since additional $\mathrm{U}(1)$ symmetries have been widely used to stabilize the dark mater candidates.

\subsection{Non-standard interactions}

NSIs in $\nu$-e scatting are usually formulated by the following Lagrangian:

$$
\mathcal{L}_{\text {NSI }} \supset 2 \sqrt{2} G_{F} \bar{\nu}_{\alpha} \gamma^{\mu} P_{L} \nu_{\beta} \overline{\psi_{e}} \gamma^{\mu}\left[\epsilon_{\alpha \beta}^{L} P_{L}+\epsilon_{\alpha \beta}^{R} P_{R}\right] \psi_{e},
$$

where $\psi_{e}$ is the Dirac spinor of the electron, $\epsilon_{\alpha \beta}^{L}$ and $\epsilon_{\alpha \beta}^{R}$ are flavor-dependent constants, and $P_{L / R} \equiv\left(1 \mp \gamma^{5}\right) / 2$. This Lagrangian originates in some models with heavy vector bosons that interact with neutrinos and electrons (see, e.g., [85]). In addition, purely scalar interactions can also generate NSIs at the loop level, and these loop-induced NSI could potentially be very large in the presence of some secret neutrino-scalar interactions [86].

Including NSIs, the cross section of $\nu_{e^{-}} e$ scattering can be derived by summing the cross sections of the three processes $\nu_{e}+e \rightarrow \nu_{\alpha}+e$, where $\alpha=e, \mu$ or $\tau$, which is

$$
\frac{d \sigma}{d T}=\frac{m_{e} G_{F}^{2}}{2 \pi}\left[\tilde{g}_{1}^{2}+\tilde{g}_{2}^{2}\left(1-\frac{T}{E_{\nu}}\right)^{2}-x_{12} \frac{m_{e} T}{E_{\nu}^{2}}\right],
$$




\begin{tabular}{|cccc|}
\hline & $\epsilon_{e e}^{P}$ & $\epsilon_{e \mu}^{P}$ & $\epsilon_{e \tau}^{P}$ \\
\hline known bounds $(P=L)$ & {$[-0.021,0.052]$} & {$[-0.13,0.13]$} & {$[-0.33,0.33]$} \\
known bounds $(P=R)$ & {$[-0.07,0.08]$} & {$[-0.13,0.13]$} & {$[-0.19,0.19]$} \\
\hline Configure A $(P=L)$ & {$[-0.017,0.015]$} & {$[-0.14,0.14]$} & {$[-0.14,0.14]$} \\
Configure A $(P=R)$ & {$[-0.10,0.08]$} & {$[-0.17,0.17]$} & {$[-0.17,0.17]$} \\
\hline Configure B $(P=L)$ & {$[-0.009,0.009]$} & {$[-0.12,0.12]$} & {$[-0.12,0.12]$} \\
Configure B $(P=R)$ & {$[-0.06,0.05]$} & {$[-0.16,0.16]$} & {$[-0.16,0.16]$} \\
\hline Configure C $(P=L)$ & {$[-0.006,0.006]$} & {$[-0.09,0.09]$} & {$[-0.09,0.09]$} \\
Configure C $(P=R)$ & {$[-0.040,0.036]$} & {$[-0.13,0.13]$} & {$[-0.13,0.13]$} \\
\hline
\end{tabular}

Table 3. Current NSI bounds from ref. [3] compared with future ${ }^{51} \mathrm{Cr}-\mathrm{LX}$ bounds (Configures A, $\mathrm{B}$, and $\mathrm{C})$.

with $\tilde{g}_{1}^{2}, \tilde{g}_{1}^{2}$ and $x_{12}$ defined as

$$
\begin{aligned}
\tilde{g}_{1}^{2} & \equiv\left(g_{V}+g_{A}+2 \epsilon_{e e}^{L}\right)^{2}+\sum_{\beta=\mu, \tau}\left(2 \epsilon_{e \beta}^{L}\right)^{2}, \\
\tilde{g}_{2}^{2} & \equiv\left(g_{V}-g_{A}+2 \epsilon_{e e}^{R}\right)^{2}+\sum_{\beta=\mu, \tau}\left(2 \epsilon_{e \beta}^{R}\right)^{2}, \\
x_{12} & \equiv\left(g_{V}+g_{A}+2 \epsilon_{e e}^{L}\right)\left(g_{V}-g_{A}+2 \epsilon_{e e}^{R}\right)+\sum_{\beta=\mu, \tau}\left(2 \epsilon_{e \beta}^{L}\right)\left(2 \epsilon_{e \beta}^{R}\right) .
\end{aligned}
$$

Note that there is no interference between the three processes because they have different flavor neutrinos in the final state. But for $\alpha=e$, there is interference between the NSI and the SM interactions, which significantly enhances the sensitivity to $\epsilon_{e e}^{L}$ and $\epsilon_{e e}^{R}$.

In figure 4 , we present the result of a $\chi^{2}$-fit analysis on the NSI sensitivity. The $\chi^{2}$-fit is performed each time for a pair of $\left(\epsilon_{e \alpha}^{L}, \epsilon_{e \alpha}^{R}\right)$ with the other $\varepsilon^{\prime}$ 's set to zero. Because the cross section is completely symmetric under the $\mu-\tau$ exchange, $\nu_{e^{-}} e$ scattering should have exactly the same sensitivities to $\left(\epsilon_{e \mu}^{L}, \epsilon_{e \mu}^{R}\right)$ and to $\left(\epsilon_{e \tau}^{L}, \epsilon_{e \tau}^{R}\right)$. So they are shown in the same plot in the right panel. As we can see, $\left(\epsilon_{e e}^{L}, \epsilon_{e e}^{R}\right)$ would be stringently constrained by the ${ }^{51}$ Cr-LXe experiment while $\left(\epsilon_{e \mu}^{L}, \epsilon_{e \mu}^{R}\right)$ and to $\left(\epsilon_{e \tau}^{L}, \epsilon_{e \tau}^{R}\right)$ would be less constrained due to the lack of interference.

The ${ }^{51} \mathrm{Cr}-\mathrm{LX}$ bounds are compared to the current known bounds on NSIs. In general, direct measurements of neutrino-electron scattering and measurements of neutrino oscillation are sensitive to the NSIs considered here. In particular, there have been bounds from LSND and TEXONO that can be readily superposed on our results, as shown in figure 4 . As for other bounds, we refer to ref. [3] for the recently updated summary, which have been included in table 3 . We conclude that for most NSIs parameters, the ${ }^{51} \mathrm{Cr}$-LXe sensitivity to NSIs would generally exceed the current known bounds.

\subsection{SPVAT interactions}

More generally one can adopt an effective field theory (EFT) approach to study BSM neutrino interactions and write down all the possible Lorentz invariant operators as follows:

$$
\mathcal{L} \supset \frac{G_{F}}{\sqrt{2}} \sum_{a=S, P, V, A, T} \bar{\nu} \Gamma^{a} \nu\left[\overline{\psi_{e}} \Gamma^{a}\left(C_{a}+D_{a} i_{a} \gamma^{5}\right) \psi_{e}\right]
$$


with

$$
\left(\Gamma^{S}, \Gamma^{P}, \Gamma^{V}, \Gamma^{A}, \Gamma^{T}\right) \equiv\left(\mathbf{1}, i \gamma^{5}, \gamma^{\mu}, \gamma^{\mu} \gamma^{5}, \sigma^{\mu \nu} \equiv \frac{i}{2}\left[\gamma^{\mu}, \gamma^{\nu}\right]\right)
$$

Here $C_{a}$ and $D_{a}$ are real constants if $i_{a}=i$ for $a=S, P$ and $T$; and $i_{a}=1$ for $a=V$ and $A$. It should be noted that this reduces to the SM Lagrangian when $\left(C_{V}, D_{V}, C_{A}, D_{A}\right)=$ $\left(g_{V},-g_{A}, g_{A},-g_{V}\right)$ and all other $C_{a}$ and $D_{a}$ are zero.

Regarding theoretical motivations, the first four types ( $a=S, P, V$ and $A$ ) could originate from integrating out some heavy scalar or vector mediators. The tensor interactions could be generated by integrating out heavy charged scalar mediators following necessary the Fierz transformations [88]. For Dirac neutrinos, all the SPVAT interactions could exist with 10 free parameters $\left(C_{a}, D_{a}\right)$ in eq. (4.6). For Majorana neutrinos, there are further constraints on the SPVAT interactions such that the allowed parameter space is actually smaller, containing only 6 free parameters [33]. Such a difference could be used to distinguish between Dirac and Majorana neutrinos [89]. In this work, we will ignore the additional constraints of Majorana neutrinos and simply take the full parameter spaces containing 10 parameters into consideration.

In this model space, the cross section of $\nu_{e^{-}} e$ scattering is given as follows [33]:

$$
\frac{d \sigma}{d T}=\frac{m_{e} G_{F}^{2}}{2 \pi}\left[x_{1}+2 x_{2}\left(1-\frac{T}{E_{\nu}}\right)+x_{3}\left(1-\frac{T}{E_{\nu}}\right)^{2}+x_{4} \frac{M T}{4 E_{\nu}^{2}}\right],
$$

where

$$
\begin{aligned}
x_{1} \equiv & \frac{1}{4}\left(C_{A}-D_{A}+C_{V}-D_{V}\right)^{2}+\frac{1}{2} C_{P} C_{T}+\frac{1}{8}\left(C_{P}^{2}+C_{S}^{2}+D_{P}^{2}+D_{S}^{2}\right) \\
& -\frac{1}{2} C_{S} C_{T}+C_{T}^{2}+\frac{1}{2} D_{P} D_{T}-\frac{1}{2} D_{S} D_{T}+D_{T}^{2}, \\
x_{2} \equiv & -\frac{1}{8}\left(C_{P}^{2}+C_{S}^{2}+D_{P}^{2}+D_{S}^{2}\right)+C_{T}^{2}+D_{T}^{2}, \\
x_{3} \equiv & \frac{1}{4}\left(C_{A}+D_{A}-C_{V}-D_{V}\right)^{2}-\frac{1}{2} C_{P} C_{T}+\frac{1}{8}\left(C_{P}^{2}+C_{S}^{2}+D_{P}^{2}+D_{S}^{2}\right) \\
& +\frac{1}{2} C_{T} C_{S}+C_{T}^{2}-\frac{1}{2} D_{P} D_{T}+\frac{1}{2} D_{S} D_{T}+D_{T}^{2}, \\
x_{4} \equiv & -\left(C_{V}-D_{A}\right)^{2}+\left(C_{A}-D_{V}\right)^{2}+C_{S}^{2}-4 C_{T}^{2}+D_{P}^{2}-4 D_{T}^{2} .
\end{aligned}
$$

In our analysis we are interested in extracting the non-SM part, so in our $\chi^{2}$-fit we define

$$
\left(\delta C_{a}, \delta D_{a}\right) \equiv\left(C_{a}, D_{a}\right)-\left(C_{a}, D_{a}\right)^{\mathrm{SM}}
$$

and make a 2-parameter fit for each pair of $\left(\delta C_{a}, \delta D_{a}\right)$. The result is presented in figure 5 . For $a=S$ or $P$, the ${ }^{51} \mathrm{Cr}$-LXe experiment would reach sensitivity around $0.5 \sim 2$. For the other cases, the measurement could be even more precise. Note that for $a=V$ or $A$, there is parameter degeneracy which would be removed in configuration C. The situation is similar to figure 3, with the reason explained at the end of section 3. 

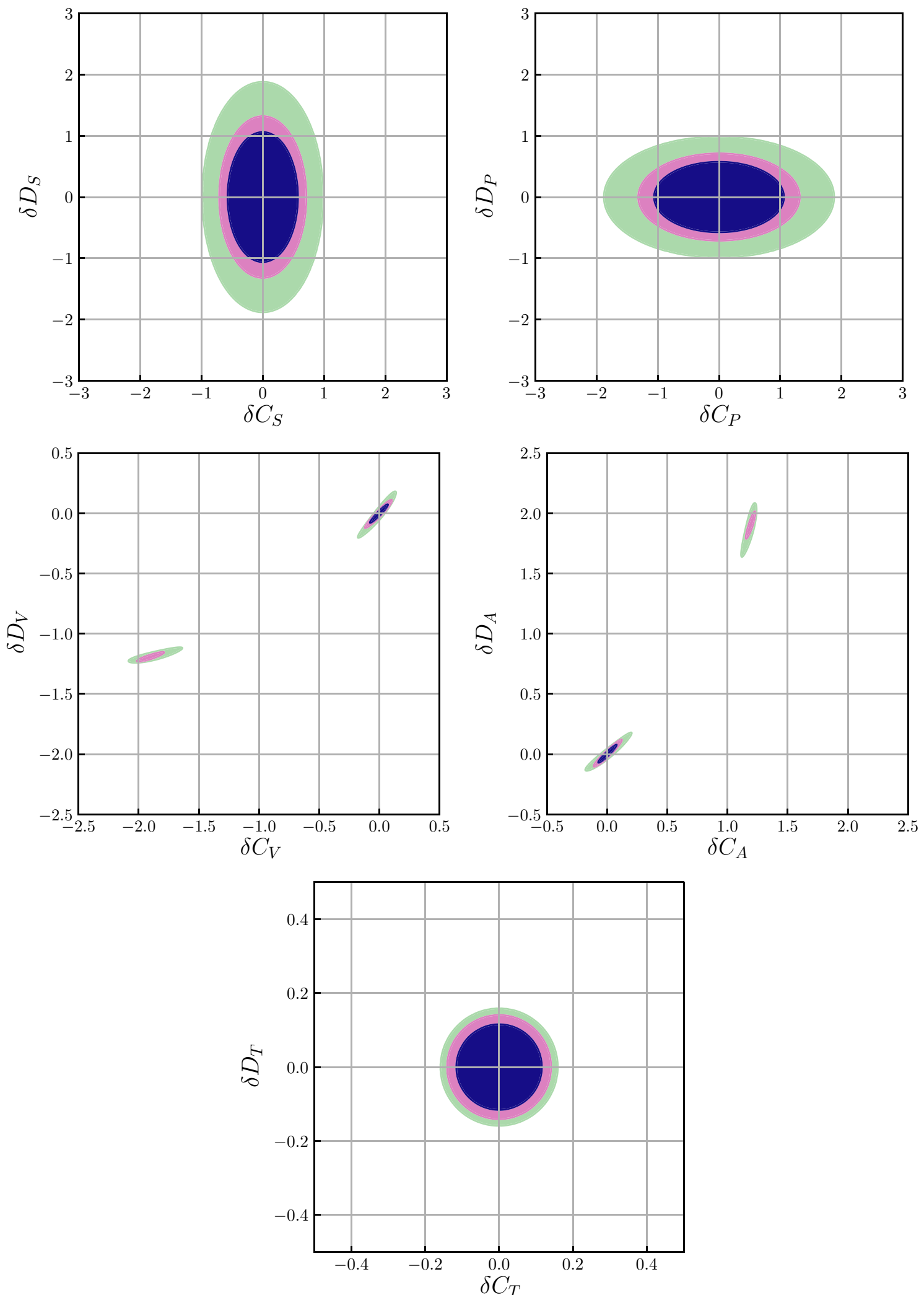

Figure 5. Constraints on the SPVAT parameters. The color coding is the same as figure 4. 


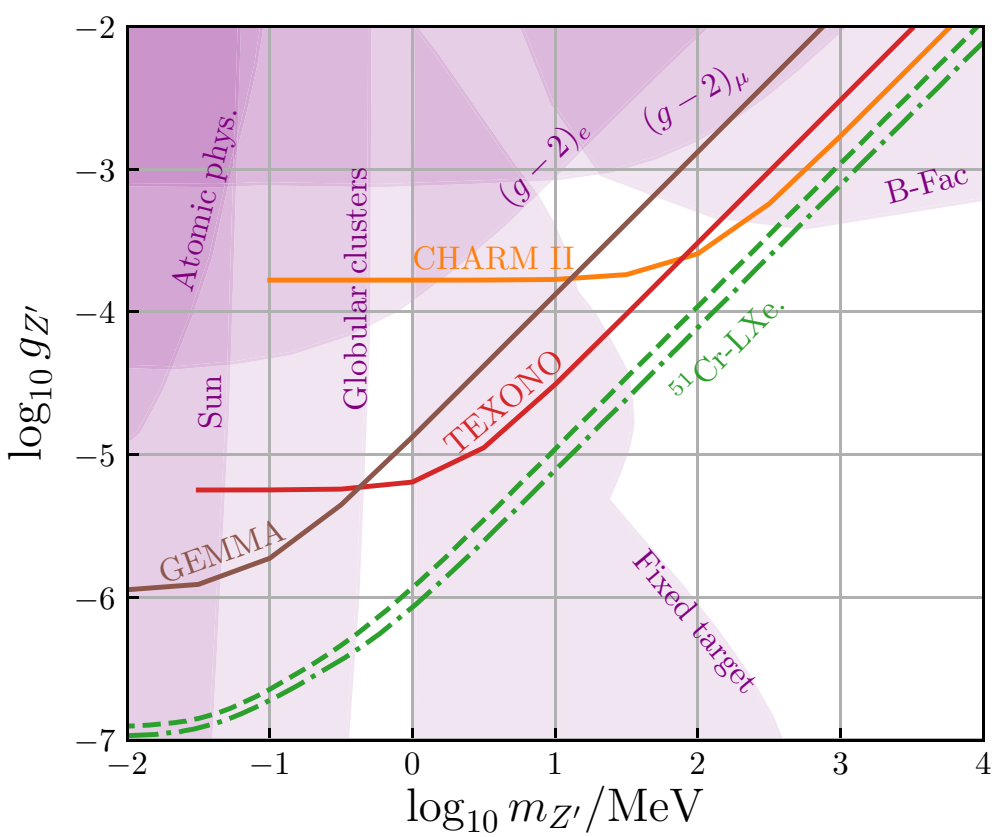

Figure 6. Constraints on the gauge coupling of light $Z^{\prime}$. The constraints of TEXONO, CHARM II, and GEMMA are taken from ref. [34], other constraints are taken from refs. [49, 90]. The green dashed curve is for ${ }^{51} \mathrm{Cr}$-LXe with configuration $\mathrm{A}$, and dot-dashed for configuration $\mathrm{C}$. Configuration B is not shown here for simplicity, which should be between the dashed and dotdashed curves.
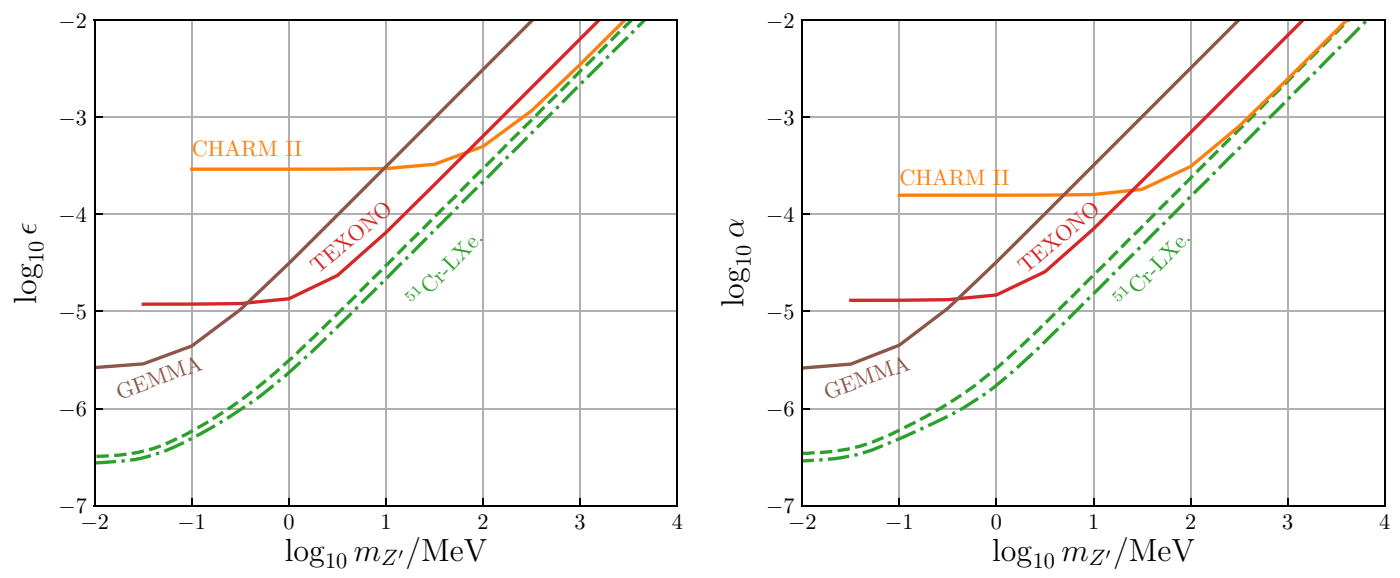

Figure 7. Constraints on the mass mixing $\alpha$ and kinetic mixing $\epsilon$ of light $Z^{\prime}$. The constraints of TEXONO, CHARM II, and GEMMA are taken from ref. [34]. The green dashed curve is for ${ }^{51} \mathrm{Cr}$-LXe with configuration A, and dot-dashed for configuration C. Configuration B is not shown here for simplicity, which should be between the dashed and dot-dashed curves.

\subsection{Light $Z^{\prime}$}

Recently a generic light neutral vector boson (commonly referred to as light $Z^{\prime}$ ) has received extensive research interest due to its connection with dark matter [73-84]. Elastic neutrino scattering is intrinsically sensitive to light mediators which usually show effect only in soft (low-momentum transfer) scattering processes, where, for most other particles, the 
electromagnetic force would be an overwhelming background. Due to their very weak SM interaction, neutrino scattering makes a very clean environment for probing new forces mediated by other light mediators [34, 44, 68, 71, 90-94].

The Lagrangian of light $Z^{\prime}$ that concerns $\nu$-e scattering is generally formulated as follows

$$
\mathcal{L} \supset-\frac{g_{Z^{\prime}}}{2} Z_{\mu}^{\prime}\left[\bar{\nu} \gamma^{\mu} \nu+\bar{\ell} \gamma^{\mu} \ell\right]-\frac{1}{2} m_{Z^{\prime}}^{2} Z_{\mu}^{\prime} Z^{\prime \mu},
$$

where $g_{Z^{\prime}}$ is the gauge coupling and $\ell$ is a charged lepton. Depending on the models, there could be different charge assignments. Here we simply assume the charge assignments are the same as the $\mathrm{U}(1)_{B-L}$ model (i.e., all leptons have the same charge). In generic $Z^{\prime}$ models, the $Z^{\prime}$ boson has both kinetic mixing and mass mixing with the SM $Z^{0}$ boson. As it has been studied in ref. [64], in the presence of both mixing, the $Z^{\prime}$ and $Z^{0}$ interactions can be formulated as

$$
\mathcal{L} \supset J_{Z}^{\mu} Z_{\mu}+J_{Z^{\prime}}^{\mu} Z_{\mu}^{\prime},
$$

where $J_{Z}^{\mu}$ and $J_{Z^{\prime}}^{\mu}$ are mixtures of the SM neutral current $J_{\mathrm{NC}}$ and a new current $J_{X}$ :

$$
\begin{aligned}
J_{Z}^{\mu} & =c_{\alpha} J_{\mathrm{NC}}^{\mu}-s_{\alpha} J_{X}^{\mu}, \\
J_{Z^{\prime}}^{\mu} & =s_{\alpha} J_{\mathrm{NC}}^{\mu}+c_{\alpha} J_{X}^{\mu} .
\end{aligned}
$$

Here $\alpha$ is the mass mixing angle with $\left(c_{\alpha}, s_{\alpha}\right) \equiv(\cos \alpha, \sin \alpha)$. The leptonic part of the $\mathrm{SM}$ neutral current $J_{\mathrm{NC}}^{\mu}$ is

$$
J_{\mathrm{NC}}^{\mu}=\frac{g}{c_{W}}\left[\bar{\nu}_{L} \gamma^{\mu} \frac{1}{2} \bar{\nu}_{L}+\bar{e}_{L} \gamma^{\mu} \frac{2 s_{W}^{2}-1}{2} \bar{e}_{L}+\bar{e}_{R} \gamma^{\mu} s_{W}^{2} \bar{e}_{R}+\cdots\right],
$$

while the explicit form of $J_{X}^{\mu}$ is given by [64]:

$$
\begin{aligned}
J_{X}^{\mu}=\frac{1}{2 c_{W} \sqrt{1-\epsilon^{2}}}\left[\bar{\nu} \gamma^{\mu}\right. & \left.-c_{W} g_{Z^{\prime}}\right) \bar{\nu}+\bar{\nu}_{L} \gamma^{\mu}\left(g \epsilon s_{W}\right) \bar{\nu}_{L} \\
& \left.+\bar{e} \gamma^{\mu}\left(-c_{W} g_{Z^{\prime}}+g \epsilon s_{W}\right) \bar{e}+\bar{e}_{R} \gamma^{\mu}\left(g \epsilon s_{W}\right) \bar{e}_{R}+\cdots\right] .
\end{aligned}
$$

The kinetic mixing $\epsilon$ is defined as $L \supset-\frac{1}{2} \epsilon F^{\mu \nu} F_{\mu \nu}^{\prime}$ where $F^{\mu \nu}$ and $F_{\mu \nu}^{\prime}$ are the gauge field tensors of the $\mathrm{SM} \mathrm{U}(1)_{Y}$ and the new $\mathrm{U}(1)$ respectively.

The contribution of $Z^{\prime}$ to the $\nu_{e}-e$ cross section can be included by adding an energydependent term to $\left(g_{1}, g_{2}\right)$ in eq. (3.5):

$$
\left(g_{1}, g_{2}\right) \rightarrow\left(g_{1}, g_{2}\right)+\frac{\left(\delta_{1}, \delta_{2}\right)}{4 \sqrt{2} G_{F}\left(2 m_{e} T+m_{Z^{\prime}}^{2}\right)},
$$

where $\left(\delta_{1}, \delta_{2}\right)$ depends on $g_{Z^{\prime}}, \epsilon$ and $\alpha$. When studying the effect of one of these three parameters, we assume the other two are negligibly small. Under this assumption, the expressions of $\left(\delta_{1}, \delta_{2}\right)$ are given as follows:

$$
\begin{array}{ll}
\left(\delta_{1}, \delta_{2}\right)=\left(g_{Z^{\prime}}^{2}, g_{Z^{\prime}}^{2}\right), & \text { for } g_{Z^{\prime}} \neq 0, \epsilon=\alpha=0, \\
\left(\delta_{1}, \delta_{2}\right)=\left(\frac{g^{2} s_{W}^{2} \epsilon^{2}}{c_{W}^{2}\left(1-\epsilon^{2}\right)}, \frac{2 g^{2} s_{W}^{2} \epsilon^{2}}{c_{W}^{2}\left(1-\epsilon^{2}\right)}\right), & \text { for } \epsilon \neq 0, g_{Z^{\prime}}=\alpha=0, \\
\left(\delta_{1}, \delta_{2}\right)=\left(\frac{g^{2} s_{\alpha}^{2}\left(2 s_{W}^{2}-1\right)}{c_{W}^{2}}, \frac{2 g^{2} s_{\alpha}^{2} s_{W}^{2}}{c_{W}^{2}}\right), & \text { for } \alpha \neq 0, g_{Z^{\prime}}=\epsilon=0 .
\end{array}
$$


Using eqs. (4.20) to (4.23), we study the sensitivity of the Cr-LXe experiment to the three parameters $g_{Z^{\prime}}, \epsilon, \alpha$ and present the results in figures 6 and 7 , in comparison with other known bounds taken from refs. [34, 49, 90]. For the gauge coupling $g_{Z^{\prime}}$ shown in figure 6, in general, neutrino-electron scattering experiments (CHARM II, TEXONO, and GEMMA) provide the leading constraints prior to some constraints obtained from atomic physics and measurements of muon and electron anomalous magnetic moments, $(g-2)_{e}$ and $(g-2)_{\mu}$. They are also complementary to some other constraints from fixed target experiments, astrophysical observations (energy loss in the sun and globular clusters), and the B-Factories. Due to its low threshold and high statistics, the ${ }^{51} \mathrm{Cr}-\mathrm{LXe}$ experiment would significantly improve upon existing constraints from neutrino-electron scattering. In the low mass limit, the ${ }^{51} \mathrm{Cr}$-LXe experiment has sensitivity to small $g_{Z^{\prime}}$ down to $10^{-7}$. For heavy $Z^{\prime}$, it would exceed the CHARM II experiment which is based on high-energy neutrino beams. The bounds on the kinetic mixing $\epsilon$ and the mass mixing $\alpha$ in figure 7 are similar to the $g_{Z^{\prime}}$ bounds. Even with configuration A, the ${ }^{51} \mathrm{Cr}$-LXe experiment could provide the most stringent constraints on all these $Z^{\prime}$ parameters, showing its the great potential in constraining or probing the new physics of light $Z^{\prime}$.

\section{Conclusion}

In this work, we have studied the potential for $\nu_{e}-e$ scattering in a LXe dark matter detector using ${ }^{51} \mathrm{Cr}$ as a radioactive neutrino source $\left({ }^{51} \mathrm{Cr}\right.$-LXe). Assuming a $5 \mathrm{MCi}{ }^{51} \mathrm{Cr}$ source located $1 \mathrm{~m}$ below a 6 -ton LXe detector with the current state-of-the-art background and running for 100 days (configuration A), one can already achieve a high statistics $(1.2 \times$ $10^{4}$ signal events) measurement of $\nu_{e}-e$ scattering. However, only the low energy part $(T \lesssim 100 \mathrm{keV}$ ) of the recoil spectrum can be efficiently measured while the higher energy signal would be submerged by the background, as shown in figure 1 . To enhance the signal/background ratio at high $T$ so that the full recoil spectrum $(0<T \lesssim 559 \mathrm{keV})$ can be measured, the background must be reduced by, for example, using ${ }^{136} \mathrm{Xe}$-depleted LXe and a shorter exposure time. For this purpose, we also considered configurations $\mathrm{B}$ and $\mathrm{C}$ (see table 1) for which the signal is greater than the background over the whole spectrum (see figure 1).

Based on these three configurations, we study low energy precision measurement of the SM parameters $\sin ^{2} \theta_{W}, g_{V}$ and $g_{A}$. For $\sin ^{2} \theta_{W}$, we find that $1 \sim 2 \%$ precision can be attained, as presented in figure 2, and table 2. As for $g_{V}$ and $g_{A}$ ), such an experiment would provide the most precise measurement among $\stackrel{(-)}{\nu}$ scattering experiments (see figure 3 ), and be comparable to the CHARM II measurements based on $\stackrel{(-)}{\nu}$ scattering. Due to this high precision, we expect that the ${ }^{51} \mathrm{Cr}$-LXe experiment would be excellent at constraining BSM neutrino interactions. Indeed, according to our analyses, it would generate leading or complementary constraints on NSIs, SPVAT interactions, and light $Z^{\prime}$, as presented in figures 4, 5, and 6 respectively. For example, take NSIs, the most extensively studied scenario: the ${ }^{51} \mathrm{Cr}$-LXe experiment is sensitive to six of the NSI parameters, namely $\epsilon_{e e}^{L}$, $\epsilon_{e e}^{R}, \epsilon_{e \mu}^{L}, \epsilon_{e \mu}^{R}, \epsilon_{e \tau}^{L}$, and $\epsilon_{e \tau}^{R}$. In table 3 and figure 4 we show that $\epsilon_{e \mu}^{L}, \epsilon_{e \mu}^{R}, \epsilon_{e \tau}^{L}$, and $\epsilon_{e \tau}^{R}$ would be constrained to the limit around $0.1 \sim 0.2$, and $\epsilon_{e e}^{L}$ and $\epsilon_{e e}^{R}$ can be more stringently 
constrained due to the interference with the SM signal, sometimes even down to $6 \times 10^{-3}$. These constraints would generally exceed the existing bounds, which would be important for future long baseline experiments such as DUNE and T2HK. In addition, probing SPVAT interactions and light $Z^{\prime}$ in $\nu_{e}-e$ scattering would shed light on the fundamental theories of both neutrinos and dark matter.

\section{Acknowledgments}

We thank Werner Rodejohann for helpful discussions. This work was supported by the Max Planck Gesellschaft (MPG), and the U.S. Department of Energy under grant number DE-SC0018327.

\section{A Geometrical calculation of $r_{\text {avg }}$}

According to the definition of $r_{\text {avg }}$ in eq. (2.6), we have

$$
\frac{1}{r_{\mathrm{avg}}^{2}}=\frac{1}{V} \int d V \frac{1}{r^{2}}=\frac{1}{V} \int \frac{1}{r^{2}} d x d y d z
$$

where the volume is

$$
V=\pi(d / 2)^{2} h
$$

Here the Cartesian coordinate $x-y-z$ is set in such a way that $z$ is the cylindrical axis the $x-y$ is the horizontal plane. By defining

$$
R^{2} \equiv x^{2}+y^{2},
$$

we can compute the integral in the cylindrical coordinate system:

$$
\begin{aligned}
\int \frac{1}{r^{2}} d x d y d z= & \int \frac{R}{(R \cos \theta)^{2}+(R \sin \theta)^{2}+z^{2}} d z d R d \theta \\
= & \int_{0}^{d / 2} d R \int_{L}^{L+h} \frac{2 \pi R}{R^{2}+z^{2}} d z \\
= & \pi d \tan ^{-1}\left(\frac{2(h+L)}{d}\right)-\pi d \tan ^{-1}\left(\frac{2 L}{d}\right) \\
& +\pi L \log \frac{L^{2}\left(d^{2}+4(h+L)^{2}\right)}{\left(d^{2}+4 L^{2}\right)(h+L)^{2}}+\pi h \log \frac{d^{2}+4(h+L)^{2}}{4(h+L)^{2}} .
\end{aligned}
$$

Therefore, the result is

$$
\begin{aligned}
r_{\mathrm{avg}}=\left[\frac{4 L}{d^{2} h} \log \frac{L^{2}\left(d^{2}+4(h+L)^{2}\right)}{\left(d^{2}+4 L^{2}\right)(h+L)^{2}}+\frac{4}{d^{2}} \log \frac{d^{2}+4(h+L)^{2}}{4(h+L)^{2}}\right. \\
\left.\quad-\frac{4}{d h} \tan ^{-1}\left(\frac{2 L}{d}\right)+\frac{4}{d h} \tan ^{-1}\left(\frac{2(h+L)}{d}\right)\right]^{-\frac{1}{2}} .
\end{aligned}
$$

Open Access. This article is distributed under the terms of the Creative Commons Attribution License (CC-BY 4.0), which permits any use, distribution and reproduction in any medium, provided the original author(s) and source are credited. 


\section{References}

[1] S. Davidson, C. Pena-Garay, N. Rius and A. Santamaria, Present and future bounds on nonstandard neutrino interactions, JHEP 03 (2003) 011 [hep-ph/0302093] [INSPIRE].

[2] T. Ohlsson, Status of non-standard neutrino interactions, Rept. Prog. Phys. 76 (2013) 044201 [arXiv: 1209.2710] [INSPIRE].

[3] Y. Farzan and M. Tortola, Neutrino oscillations and non-standard interactions, Front. Phys. 6 (2018) 10 [arXiv:1710.09360].

[4] I. Esteban, M.C. Gonzalez-Garcia, M. Maltoni, I. Martinez-Soler and J. Salvado, Updated Constraints on Non-Standard Interactions from Global Analysis of Oscillation Data, JHEP 08 (2018) 180 [arXiv: 1805.04530] [INSPIRE].

[5] D.V. Forero and P. Huber, Hints for leptonic CP-violation or New Physics?, Phys. Rev. Lett. 117 (2016) 031801 [arXiv:1601.03736] [INSPIRE].

[6] O.G. Miranda, M. Tortola and J.W.F. Valle, New ambiguity in probing CP-violation in neutrino oscillations, Phys. Rev. Lett. 117 (2016) 061804 [arXiv:1604.05690] [INSPIRE].

[7] M. Masud, A. Chatterjee and P. Mehta, Probing CP-violation signal at DUNE in presence of non-standard neutrino interactions, J. Phys. G 43 (2016) 095005 [arXiv: 1510.08261] [INSPIRE].

[8] P. Bakhti and Y. Farzan, CP-violation and non-standard interactions at the MOMENT, JHEP 07 (2016) 109 [arXiv: 1602.07099] [INSPIRE].

[9] M. Masud and P. Mehta, Nonstandard interactions spoiling the CP-violation sensitivity at DUNE and other long baseline experiments, Phys. Rev. D 94 (2016) 013014 [arXiv: 1603.01380] [INSPIRE].

[10] S. C and R. Mohanta, Impact of lepton flavor universality violation on CP-violation sensitivity of long-baseline neutrino oscillation experiments, Eur. Phys. J. C 77 (2017) 32 [arXiv: 1701.00327] [INSPIRE].

[11] J.M. Hyde, Biprobability approach to CP phase degeneracy from non-standard neutrino interactions, arXiv:1806.09221 [INSPIRE].

[12] K.N. Deepthi, S. Goswami and N. Nath, Challenges posed by non-standard neutrino interactions in the determination of $\delta_{\mathrm{CP}}$ at DUNE, Nucl. Phys. B 936 (2018) 91 [arXiv: 1711.04840] [INSPIRE].

[13] W. Konetschny and W. Kummer, Nonconservation of total lepton number with scalar bosons, Phys. Lett. 70B (1977) 433 [INSPIRE].

[14] T.P. Cheng and L.-F. Li, Neutrino masses, mixings and oscillations in $\mathrm{SU}(2) \times \mathrm{U}(1)$ models of electroweak interactions, Phys. Rev. D 22 (1980) 2860 [InSPIRE].

[15] G. Lazarides, Q. Shafi and C. Wetterich, Proton lifetime and fermion masses in an $\mathrm{SO}(10)$ model, Nucl. Phys. B 181 (1981) 287 [INSPIRE].

[16] R.N. Mohapatra and G. Senjanović, Neutrino masses and mixings in gauge models with spontaneous parity violation, Phys. Rev. D 23 (1981) 165 [INSPIRE].

[17] J. Schechter and J.W.F. Valle, Neutrino masses in $\mathrm{SU}(2) \times \mathrm{U}(1)$ theories, Phys. Rev. D 22 (1980) 2227 [INSPIRE]. 
[18] J. Schechter and J.W.F. Valle, Neutrino decay and spontaneous violation of lepton number, Phys. Rev. D 25 (1982) 774 [INSPIRE].

[19] J.C. Pati and A. Salam, Lepton number as the fourth color, Phys. Rev. D 10 (1974) 275 [Erratum ibid. D 11 (1975) 703] [INSPIRE].

[20] R.N. Mohapatra and J.C. Pati, A natural left-right symmetry, Phys. Rev. D 11 (1975) 2558 [INSPIRE].

[21] G. Senjanović and R.N. Mohapatra, Exact left-right symmetry and spontaneous violation of parity, Phys. Rev. D 12 (1975) 1502 [InSPIRE].

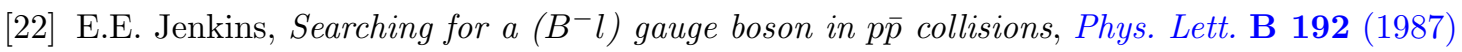
219 [INSPIRE].

[23] X.G. He, G.C. Joshi, H. Lew and R.R. Volkas, NEW Z' phenomenology, Phys. Rev. D 43 (1991) 22 [INSPIRE].

[24] W. Buchmüller, C. Greub and P. Minkowski, Neutrino masses, neutral vector bosons and the scale of B-L breaking, Phys. Lett. B 267 (1991) 395 [INSPIRE].

[25] X.-G. He, G.C. Joshi, H. Lew and R.R. Volkas, Simplest Z' model, Phys. Rev. D 44 (1991) 2118 [INSPIRE].

[26] R. Foot, X.G. He, H. Lew and R.R. Volkas, Model for a light $Z^{\prime}$ boson, Phys. Rev. D 50 (1994) 4571 [hep-ph/9401250] [INSPIRE].

[27] W. Emam and S. Khalil, Higgs and $Z^{\prime}$ phenomenology in B-L extension of the standard model at LHC, Eur. Phys. J. C 52 (2007) 625 [arXiv:0704.1395] [InSPIRE].

[28] L. Basso, A. Belyaev, S. Moretti and C.H. Shepherd-Themistocleous, Phenomenology of the minimal B-L extension of the Standard model: $Z^{\prime}$ and neutrinos, Phys. Rev. D 80 (2009) 055030 [arXiv: 0812 .4313] [INSPIRE].

[29] B. Sevda et al., Constraints on scalar-pseudoscalar and tensorial non-standard interaction and tensorial unparticle couplings from neutrino-electron scattering, Phys. Rev. D 95 (2017) 033008 [arXiv: 1611.07259] [INSPIRE].

[30] TEXONO collaboration, Measurement of $\nu_{e}$-bar electron scattering cross-section with a CsI(Tl) scintillating crystal array at the Kuo-Sheng nuclear power reactor, Phys. Rev. D 81 (2010) 072001 [arXiv:0911.1597] [INSPIRE].

[31] D.V. Forero and M.M. Guzzo, Constraining nonstandard neutrino interactions with electrons, Phys. Rev. D 84 (2011) 013002 [INSPIRE].

[32] Y. Kaneta and T. Shimomura, On the possibility of a search for the $L_{\mu}-L_{\tau}$ gauge boson at Belle-II and neutrino beam experiments, PTEP 2017 (2017) 053B04 [arXiv:1701.00156] [INSPIRE].

[33] W. Rodejohann, X.-J. Xu and C.E. Yaguna, Distinguishing between Dirac and Majorana neutrinos in the presence of general interactions, JHEP 05 (2017) 024 [arXiv:1702.05721] [INSPIRE].

[34] M. Lindner, F.S. Queiroz, W. Rodejohann and X.-J. Xu, Neutrino-electron scattering: general constraints on $Z^{\prime}$ and dark photon models, JHEP 05 (2018) 098 [arXiv: 1803.00060] [INSPIRE].

[35] I. Bischer and W. Rodejohann, General neutrino interactions at the DUNE near detector, Phys. Rev. D 99 (2019) 036006 [arXiv: 1810.02220] [INSPIRE]. 
[36] C.A. Argüelles, M. Hostert and Y.-D. Tsai, Testing new physics explanations of MiniBooNE anomaly at neutrino scattering experiments, arXiv:1812.08768 [INSPIRE].

[37] B. Sevda et al., Constraints on nonstandard intermediate boson exchange models from neutrino-electron scattering, Phys. Rev. D 96 (2017) 035017 [arXiv:1702.02353] [INSPIRE].

[38] K.A. Kouzakov and A.I. Studenikin, Electromagnetic properties of massive neutrinos in low-energy elastic neutrino-electron scattering, Phys. Rev. D 95 (2017) 055013 [Erratum ibid. D 96 (2017) 099904] [arXiv:1703.00401] [InSPIRE].

[39] A.N. Khan and D.W. McKay, $\sin ^{2}(\theta) w$ estimate and bounds on nonstandard interactions at source and detector in the solar neutrino low-energy regime, JHEP 07 (2017) 143 [arXiv: 1704.06222] [INSPIRE].

[40] A.N. Khan, Global analysis of the source and detector nonstandard interactions using the short baseline $\nu$-e and $\nu^{-}$-e scattering data, Phys. Rev. D 93 (2016) 093019 [arXiv: 1605.09284] [INSPIRE].

[41] A.N. Khan, $\sin ^{2} \theta_{W}$ estimate and neutrino electromagnetic properties from low-energy solar data, J. Phys. G 46 (2019) 035005 [arXiv:1709.02930] [INSPIRE].

[42] K.S. Babu, A. Friedland, P.A.N. Machado and I. Mocioiu, Flavor gauge models below the Fermi scale, JHEP 12 (2017) 096 [arXiv:1705.01822] [INSPIRE].

[43] M.D. Campos et al., Neutrino masses and absence of flavor changing interactions in the 2HDM from gauge principles, JHEP 08 (2017) 092 [arXiv: 1705.05388] [INSPIRE].

[44] M. Bauer, P. Foldenauer and J. Jaeckel, Hunting all the hidden photons, JHEP 07 (2018) 094 [arXiv: 1803.05466] [INSPIRE].

[45] CHARM-II collaboration, Measurement of differential cross-sections for muon-neutrino electron scattering, Phys. Lett. B 302 (1993) 351 [INSPIRE].

[46] CHARM-II collaboration, Precision measurement of electroweak parameters from the scattering of muon-neutrinos on electrons, Phys. Lett. B 335 (1994) 246 [INSPIRE].

[47] LSND collaboration, Measurement of electron-neutrino-electron elastic scattering, Phys. Rev. D 63 (2001) 112001 [hep-ex/0101039] [INSPIRE].

[48] M. Pospelov, Neutrino physics with dark matter experiments and the signature of new baryonic neutral currents, Phys. Rev. D 84 (2011) 085008 [arXiv:1103.3261] [INSPIRE].

[49] R. Harnik, J. Kopp and P.A.N. Machado, Exploring $\nu$ signals in dark matter detectors, JCAP 07 (2012) 026 [arXiv:1202.6073] [INSPIRE].

[50] M. Pospelov and J. Pradler, Elastic scattering signals of solar neutrinos with enhanced baryonic currents, Phys. Rev. D 85 (2012) 113016 [Erratum ibid. D 88 (2013) 039904] [arXiv: 1203.0545] [INSPIRE].

[51] M. Pospelov and J. Pradler, Dark matter or neutrino recoil? Interpretation of recent experimental results, Phys. Rev. D 89 (2014) 055012 [arXiv:1311.5764] [INSPIRE].

[52] P. Coloma, P. Huber and J.M. Link, Combining dark matter detectors and electron-capture sources to hunt for new physics in the neutrino sector, JHEP 11 (2014) 042 [arXiv: 1406.4914] [INSPIRE].

[53] D.C. Malling et al., After LUX: the LZ program, arXiv:1110.0103.

[54] LZ collaboration, LUX-ZEPLIN (LZ) conceptual design report, arXiv:1509.02910. 
[55] B.J. Mount et al., LUX-ZEPLIN (LZ) technical design report, arXiv:1703.09144 [INSPIRE].

[56] M. Cribier et al., Production of a 62-PBq Cr-51 low-energy neutrino source for GALLEX, Nucl. Instrum. Meth. A 378 (1996) 233 [INSPIRE].

[57] G. Arcadi, M. Lindner, J. Martins and F.S. Queiroz, New physics probes: atomic parity violation, polarized electron scattering and neutrino-nucleus coherent scattering, arXiv:1906.04755 [INSPIRE].

[58] C. Giunti and C.W. Kim, Fundamentals of neutrino physics and astrophysics, Oxford University Press, Oxford U.K. (2007).

[59] L. Baudis et al., Neutrino physics with multi-ton scale liquid xenon detectors, JCAP 01 (2014) 044 [arXiv: 1309.7024] [INSPIRE].

[60] Particle Data Group collaboration, Review of particle physics, Phys. Rev. D 98 (2018) 030001 [INSPIRE].

[61] J. Erler and M.J. Ramsey-Musolf, The weak mixing angle at low energies, Phys. Rev. D 72 (2005) 073003 [hep-ph/0409169] [INSPIRE].

[62] J. Erler and S. Su, The weak neutral current, Prog. Part. Nucl. Phys. 71 (2013) 119 [arXiv: 1303.5522] [INSPIRE].

[63] K.J. Healey, A.A. Petrov and D. Zhuridov, Nonstandard neutrino interactions and transition magnetic moments, Phys. Rev. D 87 (2013) 117301 [Erratum ibid. D 89 (2014) 059904] [arXiv: 1305.0584] [INSPIRE].

[64] M. Lindner, W. Rodejohann and X.-J. Xu, Coherent neutrino-nucleus scattering and new neutrino interactions, JHEP 03 (2017) 097 [arXiv: 1612.04150] [INSPIRE].

[65] L. Heurtier and Y. Zhang, Supernova constraints on massive (pseudo)scalar coupling to neutrinos, JCAP 02 (2017) 042 [arXiv: 1609.05882] [INSPIRE].

[66] D.K. Papoulias and T.S. Kosmas, COHERENT constraints to conventional and exotic neutrino physics, Phys. Rev. D 97 (2018) 033003 [arXiv:1711.09773] [InSPIRE].

[67] G. Magill and R. Plestid, Probing new charged scalars with neutrino trident production, Phys. Rev. D 97 (2018) 055003 [arXiv:1710.08431] [INSPIRE].

[68] Y. Farzan, M. Lindner, W. Rodejohann and X.-J. Xu, Probing neutrino coupling to a light scalar with coherent neutrino scattering, JHEP 05 (2018) 066 [arXiv:1802.05171] [INSPIRE].

[69] Y. Yang and J.P. Kneller, Neutrino flavor transformation in supernovae as a probe for nonstandard neutrino-scalar interactions, Phys. Rev. D 97 (2018) 103018 [arXiv: 1803.04504] [INSPIRE].

[70] D. Aristizabal Sierra, V. De Romeri and N. Rojas, COHERENT analysis of neutrino generalized interactions, Phys. Rev. D 98 (2018) 075018 [arXiv:1806.07424] [INSPIRE].

[71] V. Brdar, W. Rodejohann and X.-J. Xu, Producing a new Fermion in coherent elastic neutrino-nucleus scattering: from neutrino mass to dark matter, JHEP 12 (2018) 024 [arXiv: 1810.03626] [INSPIRE].

[72] A. Błaut and W. Sobków, Neutrino elastic scattering on polarized electrons as tool for probing neutrino nature, arXiv:1812.09828 [INSPIRE].

[73] D. Feldman, Z. Liu and P. Nath, The Stueckelberg $Z^{\prime}$ extension with kinetic mixing and milli-charged dark matter from the hidden sector, Phys. Rev. D 75 (2007) 115001 [hep-ph/0702123] [INSPIRE]. 
[74] E. Dudas, Y. Mambrini, S. Pokorski and A. Romagnoni, (In)visible $Z^{\prime}$ and dark matter, JHEP 08 (2009) 014 [arXiv:0904.1745] [INSPIRE].

[75] M.R. Buckley, D. Hooper, J. Kopp and E. Neil, Light $Z^{\prime}$ bosons at the Tevatron, Phys. Rev. D 83 (2011) 115013 [arXiv:1103.6035] [InSPIRE].

[76] H. An, X. Ji and L.-T. Wang, Light dark matter and $Z^{\prime}$ dark force at colliders, JHEP 07 (2012) 182 [arXiv: 1202.2894] [INSPIRE].

[77] G. Arcadi, Y. Mambrini, M.H.G. Tytgat and B. Zaldivar, Invisible $Z^{\prime}$ and dark matter: LHC vs LUX constraints, JHEP 03 (2014) 134 [arXiv: 1401.0221] [INSPIRE].

[78] A. Alves, S. Profumo and F.S. Queiroz, The dark $Z^{\prime}$ portal: direct, indirect and collider searches, JHEP 04 (2014) 063 [arXiv: 1312.5281] [INSPIRE].

[79] J.M. Cline, G. Dupuis, Z. Liu and W. Xue, The windows for kinetically mixed Z'-mediated dark matter and the galactic center gamma ray excess, JHEP 08 (2014) 131 [arXiv:1405.7691] [INSPIRE].

[80] O. Ducu, L. Heurtier and J. Maurer, LHC signatures of a $Z^{\prime}$ mediator between dark matter and the $\mathrm{SU}(3)$ sector, JHEP 03 (2016) 006 [arXiv:1509.05615] [INSPIRE].

[81] O. Buchmueller, M.J. Dolan, S.A. Malik and C. McCabe, Characterising dark matter searches at colliders and direct detection experiments: vector mediators, JHEP 01 (2015) 037 [arXiv: 1407.8257] [INSPIRE].

[82] A. Alves, A. Berlin, S. Profumo and F.S. Queiroz, Dirac-fermionic dark matter in U(1) $X$ models, JHEP 10 (2015) 076 [arXiv:1506.06767] [INSPIRE].

[83] N. Okada and S. Okada, $Z_{\mathrm{BL}}^{\prime}$ portal dark matter and LHC Run-2 results, Phys. Rev. D 93 (2016) 075003 [arXiv:1601.07526] [INSPIRE].

[84] S.-F. Ge and I.M. Shoemaker, Constraining photon portal dark matter with Texono and coherent data, JHEP 11 (2018) 066 [arXiv:1710.10889] [INSPIRE].

[85] J. Heeck, M. Lindner, W. Rodejohann and S. Vogl, Non-standard neutrino interactions and neutral gauge bosons, SciPost Phys. 6 (2019) 038 [arXiv:1812.04067] [INSPIRE].

[86] I. Bischer, W. Rodejohann and X.-J. Xu, Loop-induced neutrino non-standard interactions, JHEP 10 (2018) 096 [arXiv: 1807.08102] [INSPIRE].

[87] TEXONO collaboration, Constraints on non-standard neutrino interactions and unparticle physics with neutrino-electron scattering at the Kuo-Sheng nuclear power reactor, Phys. Rev. D 82 (2010) 033004 [arXiv: 1006.1947] [INSPIRE].

[88] X.-J. Xu, Tensor and scalar interactions of neutrinos may lead to observable neutrino magnetic moments, Phys. Rev. D 99 (2019) 075003 [arXiv:1901.00482] [INSPIRE].

[89] S.P. Rosen, Analog of the Michel parameter for neutrino-electron scattering: a test for Majorana neutrinos, Phys. Rev. Lett. 48 (1982) 842 [InSPIRE].

[90] S. Bilmis et al., Constraints on dark photon from neutrino-electron scattering experiments, Phys. Rev. D 92 (2015) 033009 [arXiv:1502.07763] [INSPIRE].

[91] B. Dutta, R. Mahapatra, L.E. Strigari and J.W. Walker, Sensitivity to Z-prime and nonstandard neutrino interactions from ultralow threshold neutrino-nucleus coherent scattering, Phys. Rev. D 93 (2016) 013015 [arXiv:1508.07981] [INSPIRE].

[92] J.B. Dent et al., Probing light mediators at ultralow threshold energies with coherent elastic neutrino-nucleus scattering, Phys. Rev. D 96 (2017) 095007 [arXiv:1612.06350] [InSPIRE]. 
[93] Y. Cui, M. Pospelov and J. Pradler, Signatures of dark radiation in neutrino and dark matter detectors, Phys. Rev. D 97 (2018) 103004 [arXiv:1711.04531] [INSPIRE].

[94] M. Abdullah et al., Coherent elastic neutrino nucleus scattering as a probe of a $Z^{\prime}$ through kinetic and mass mixing effects, Phys. Rev. D 98 (2018) 015005 [arXiv:1803.01224] [INSPIRE]. 